Nervenarzt 2021 · 92:1302-1304 https://doi.org/10.1007/s00115-021-01125-w Angenommen: 17. März 2021

Online publiziert: 29. April 2021

(c) Der/die Autor(en) 2021

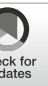

Jonathan Vöglein ${ }^{1,2} \cdot$ Johannes Levin ${ }^{1,2,3} \cdot$ Robert Forbrig ${ }^{4} \cdot$ Thomas Liebig $^{4}$. Marianne Dieterich ${ }^{1,2,3,5} \cdot$ Adrian Danek $^{1,2}$

'Neurologische Klinik und Poliklinik, LMU Klinikum, München, Deutschland

${ }^{2}$ Deutsches Zentrum für Neurodegenerative Erkrankungen (DZNE), München, Deutschland

${ }^{3}$ Munich Cluster for Systems Neurology (SyNergy), München, Deutschland

${ }^{4}$ Institut für diagnostische und interventionelle Neuroradiologie, LMU Klinikum, München, Deutschland

${ }^{5}$ Deutsches Schwindel- und Gleichgewichtszentrum (DSGZ), LMU Klinikum, München, Deutschland

\title{
Amphetamin induzierte zerebrale Vaskulitis - ein Fallbericht
}

kopfnekrose rechts unklarer Ursache erkrankt, die operativ behandelt wurde. In der Familienanamnese wurden keine Schlaganfälle berichtet. Eine relevante Dauermedikation bestand nicht, aber auf Nachfrage wurde ein Amphetaminkonsum seit 2012, zuletzt vor 2 Wochen, berichtet.

In ergänzenden MRT-Sequenzen zeigten sich im Seitenvergleich rechts ein verringertes Arterienkaliber bzw. eine fehlende Abgrenzbarkeit in der flussbasierten Darstellung der parakavernosalen und terminalen ACI sowie der gesamten A. cerebri media (๑Abb. 1b). In der „Black-blood“-Sequenz, welche flieBendes Blut durch Signalunterdrückung auch nach Kontrastmittelgabe hypointens zur Darstellung kommen lässt und deshalb besonders zur Darstellung pathologischer und segmental gesteigerter Kontrastmittelanreicherungen der Gefäßwand geeignet ist [9], stellten sich verdickte, kontrastmittelanreichernde Gefäßwände von der rechten distalen Arteria carotis interna bis in die Mediaäste der Inselregion (M2) reichend dar (• Abb. 1c).

Die Liquoruntersuchung ergab unauffällige Befunde hinsichtlich der Routineparameter, der Proteindifferenzierung, der oligoklonalen Banden und der Erregerdiagnostik. Die differenzialdiagnostische Aufarbeitung inklusive Ganzkörper FDG-PET-CT zur Abklärung eines potenziellen Gefäßbefalls im Rahmen einer systemischen Vaskulitis und zur Suche nach einer neoplastischen Erkrankung, die ein paraneoplastisches Syndrom verursachen könnte, Laboruntersuchungen (Elektrolyte, Nierenwerte, Leberwerte, Cholestaseparameter, CRP, Pankreaswerte, LDH, CK, BSG, Immunelektrophorese, Serumgesamteiweiß, Harnsäure, Differenzialblutbild, Erythrozytenindices, Hämoglobin, Hämatokrit, Gerinnungsdiagnostik, PSA, Kryoglobuline, Komplementfaktoren, Rheumafaktor, ANA, ENA, ANCA, Cardiolipin-Antikörper, $\beta$-2-GlykoproteinAntikörper, TSH, Urinstix und -sediment) und organspezifischer Diagnostik (Gastroskopie, Koloskopie, Lungenfunktionsuntersuchung, Ultraschalluntersuchung der Beinvenen, HNO-, gastroenterologische, orthopädische, dermatologische und ophthalmologische Untersuchung) zeigte keine Hinweise auf eine systemische Vaskulitis oder eine maligne Grunderkrankung. Liquor- und Blutdiagnostik lieferten keinen Anhalt für eine paraneoplastische Erkrankung. Magnetresonanztomographisch fanden sich keine weiteren Gefäß- oder Hirnparenchymläsionen, keine $\mathrm{SAB}$, keine auf eine idiopathische Moyamoya-Erkrankung hinweisende Kollateralisierung und keine Dissektion. Bei Vasospasmen, z. B. im Rahmen einer SAB oder eines RCVS, findet sich in der „Black-blood“-Sequenz typischerweise keine Kontrastmittelanreicherung der Gefäßwand. Nach 2 Wochen war der Gefäßbefund magnetresonanztomographisch unverändert. $\mathrm{Da}$ 

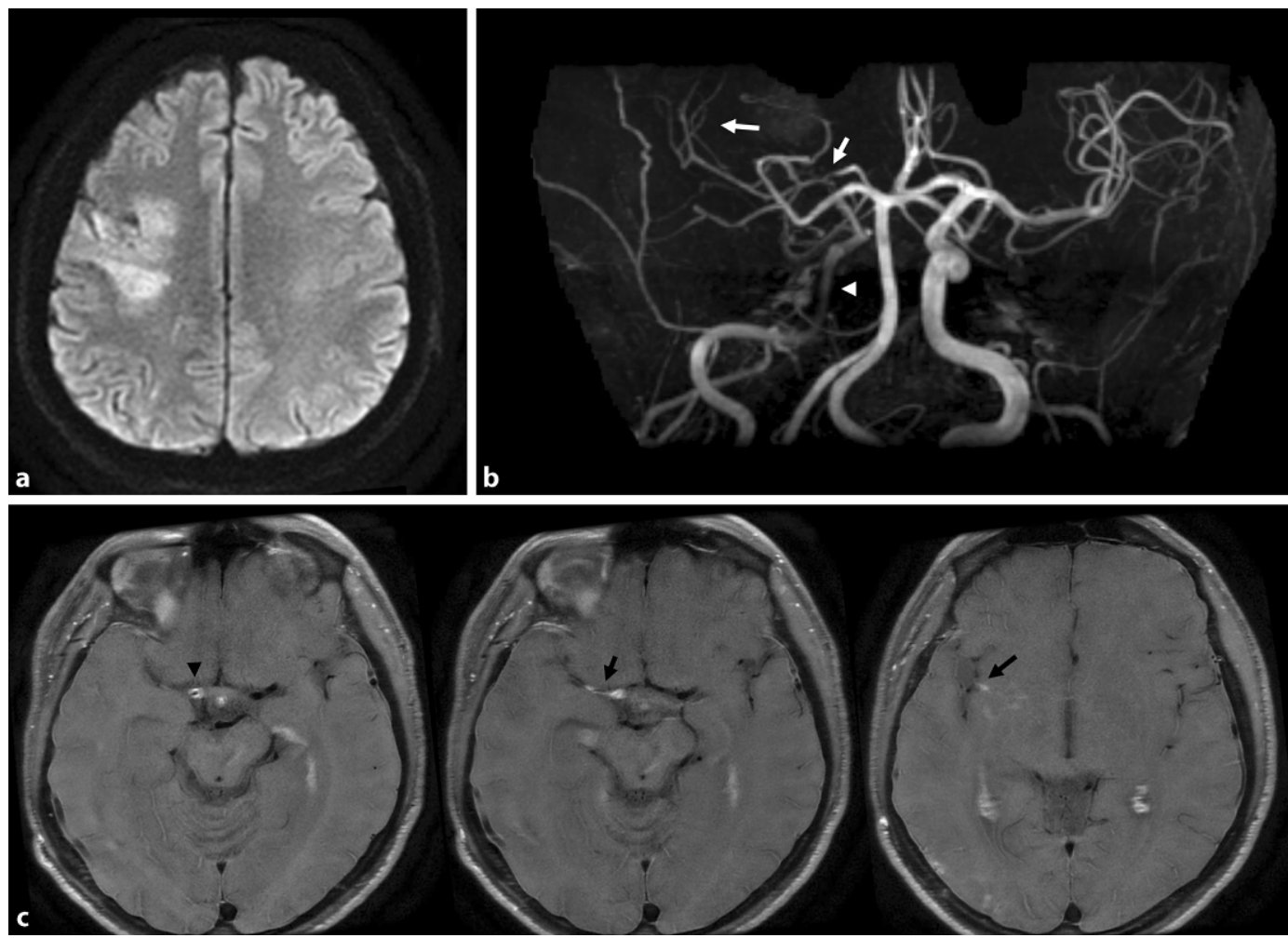

Abb. $1<$ a Mediateilinfarkt rechts in der DWI-MRT. b Im Seitenvergleich kaliberschwächere Gefäße im rechten vorderen Kreislauf beginnend im mittleren Abschnitt der Arteria carotis interna (Pfeilspitze), des Karotis-T (kurzer Pfeil) und der peripheren Mediaäste (langer Pfeil) in der TOF-Angiographie. c Langstreckig aufgetriebene und kontrastmittelanreichernde Gefäßwände im „black blood imaging" von der rechten distalen Arteria carotis interna (Pfeilspitze) über das M1-Segment (kurzer Pfeil) bis in die M2Segmente in der Inselregion (langer Pfeil) reichend

sich in der MR-Angiographie keine Hinweise auf weitere Gefäßpathologien fanden, der Befund der „Black-blood“Sequenz typisch für eine Entzündung der Gefäßwand war und sich ein klinisch stabiler Verlauf zeigte, wurde auf eine invasive Gefäßdiagnostik mittels digitaler Subtraktionsangiographie verzichtet. In der farbkodierten Duplexsonographie der extrakraniellen Arterien fand sich ein prästenotisches Flussprofil mit im Seitenvergleich reduzierten Flusswerten. Eine Arteriosklerose oder Stenosen der extrakraniellen Arterien lagen nicht vor. In der kardialen Diagnostik mittels transthorakaler Echokardiographie und automatisiertem Herzrhythmusmonitoring über $72 \mathrm{~h}$ zeigten sich keine pathologischen Befunde. Blutdruckwerte, HbAlc und LDL-Cholesterin waren unauffällig. HIV- und Hepatitis-B/CSerologien waren negativ.

Da bezüglich der Sekundärprophylaxe für den beschriebenen Einzelfall keine gesonderte Evidenz vorlag, wurde der $\mathrm{Pa}$ tient leitliniengerecht mit $100 \mathrm{mg}$ Aspirin und $40 \mathrm{mg}$ Atorvastatin täglich behandelt [8]. Es erfolgten wiederholte psychotherapeutische Kurzinterventionen mit dem Ziel einer Amphetaminabstinenz [7].
Im Verlauf kam es unter Ergotherapie zu einer Besserung der Parese und nach 2 Jahren berichtete der Patient von einer Amphetaminabstinenz seit dem Schlaganfall. Anamnestisch und klinisch fanden sich keine Hinweise auf eine erneute zerebrale Ischämie.

\section{Diskussion}

Der bildgebende Befund war ausschlaggebend für die Diagnose einer zerebralen Vaskulitis. Passend hierzu fanden sich zum Verlauf und der Lokalisation passende rechtseitige Kopfschmerzen. Die zum Zeitpunkt des Schlaganfalls 2 Jahre zurückliegende atraumatische Femurkopfnekrose, ebenfalls eine ischämische Erkrankung [1], könnte auf eine systemische Vaskulitis hindeuten. In der umfangreichen differenzialdiagnostischen Aufarbeitung fanden sich keine Hinweise auf die Ursache der zerebralen Vaskulitis, sodass der Amphetaminkonsum in Betracht kam. In der Literatur wurde von 3 Fällen mit amphetaminkonsumassoziierten ZNS-Vaskulitiden berichtet, die autoptisch gesichert wurden. Die neuropathologischen Untersuchungen zeigten nekrotisierende Gefäßwandveränderun- gen $[4,5]$. Eine Studie mit Rhesusaffen, denen für den Missbrauch beim Menschen adaptierte Amphetamindosen verabreicht wurden, zeigte in $80 \%$ der Primaten angiographische Veränderungen und Sektionsbefunde einer nekrotisierenden Vaskulitis [6]. Damit ist in diesem Fall eine amphetamininduzierte zerebrale Vaskulitis die wahrscheinlichste Diagnose. Die Evidenz für eine adäquate Therapie ist spärlich. De facto existiert nur ein Fallbericht einer Patientin mit bioptisch gesicherter amphetaminkonsumassoziierter nekrotisierender ZNS-Vaskulitis, in dem über eine Vollremission angiographischer Veränderungen im mittelfristigen Verlauf unter einer Behandlung mit Cyclophosphamid berichtet wurde [5]. Aufgrund des stabilen klinischen und bildgebenden Befundes sowie der atraumatischen Femurkopfnekrose in der Vorgeschichte, deren Hauptrisikofaktor eine Kortisontherapie darstellt [1], erfolgte bei dem Patienten keine immunsuppressive Behandlung. Interessant ist die Frage, warum nur ein Gefäßterritorium von der Vaskulitis betroffen war (oder zwei Gefäßterritorien, wenn die Femurkopfnekrose in der Vorgeschichte als Manifestation einer systemischen Vaskulitis 
Tab. 1 Potenzielle Folgen von Amphetaminkonsum auf das Gehirn und assoziierte Symptome [7]

Folge von Amphetaminkonsum

Zerebrale Ischämie

Symptome

Hirnblutungen (Subarachnoidalblutung, intrazerebrale Blutung)

Epileptische Anfälle

Psychische Symptome
Fokale neurologische Ausfälle

Donnerschlagkopfschmerz, Bewusstseinsstörung, fokale neurologische Ausfälle

Motorische Entäußerungen, Bewusstseinsstörung, Zungenbiss, Einnässen

Agitation, Stimmungsschwankungen, Aggression, psychomotorische Unruhe, Halluzinationen, paranoide Wahnvorstellungen, Panik, Abhängigkeit gesehen wird). Dies könnte der unterschiedlichen Suszeptibilität verschiedener Gewebe bzw. Territorien für pathologische Prozesse geschuldet sein. Zum anderen könnten andere subtile Vaskulitismanifestationen der Diagnostik entgangen sein. Zusammenfassend stellt die zerebrale Vaskulitis neben anderen Auswirkungen von Amphetaminen auf das zentrale Nervensystem (•Tab. 1) eine wichtige potenzielle Folge eines Amphetaminkonsums dar.

\section{Fazit für die Praxis}

- Beim Verdacht auf eine ZNS-Vaskulitis muss an eine Drogenanamnese gedacht werden.

- Amphetaminabstinenz steht therapeutisch im Vordergrund.

- Gegebenenfalls kann eine immunsuppressive Behandlung im Rahmen eines individuellen Therapiekonzeptes erfolgen.

- Die Langzeitprognose ist unklar, bei Beendigung der Amphetaminexposition möglicherweise gut.

\section{Korrespondenzadresse}

\section{Dr. Jonathan Vöglein}

Deutsches Zentrum für Neurodegenerative Erkrankungen (DZNE)

München, Deutschland

Jonathan.Voeglein@med.uni-muenchen.de

Funding. Open Access funding enabled and organized by Projekt DEAL.

\section{Einhaltung ethischer Richtlinien}

Interessenkonflikt. J. Levin legt Referentenhonorare von Roche, Bayer Vital, der Willi Gross Stiftung, Beratungshonorare von Axon Neuroscience und von lonis
Pharmaceuticals, Autorenhonorare von Thieme Medizinische Fachverlage und W. Kohlhammer GmbH Medizinische Verlage, nichtfinanzielle Unterstützung von Abbvie, eine Vergütung für Teilzeit-CMO-Tätigkeit der MODAG GmbH, alle außerhalb der eingereichten Arbeit, offen. J. Vöglein, R. Forbrig, T. Liebig, M. Dieterich und A. Danek geben an, dass kein Interessenkonflikt besteht.

Alle berichteten Daten wurden im Rahmen der klinischen Routinediagnostik gewonnen. Der Patient gab sein schriftliches Einverständnis zur Veröffentlichung der Daten. Die Ethikkommission der LMU München gab ihr Einverständnis zur Veröffentlichung des Berichtes.

Open Access. Dieser Artikel wird unter der Creative Commons Namensnennung 4.0 International Lizenz veröffentlicht, welche die Nutzung, Vervielfältigung, Bearbeitung, Verbreitung und Wiedergabe in jeglichem Medium und Format erlaubt, sofern Sie den/die ursprünglichen Autor(en) und die Quelle ordnungsgemäßnennen, einen Link zur Creative Commons Lizenz beifügen und angeben, ob Änderungen vorgenommen wurden.

Die in diesem Artikel enthaltenen Bilder und sonstiges Drittmaterial unterliegen ebenfalls der genannten Creative Commons Lizenz, sofern sich aus der Abbildungslegende nichts anderes ergibt. Sofern das betreffende Material nicht unter der genannten Creative Commons Lizenz steht und die betreffende Handlung nicht nach gesetzlichen Vorschriften erlaubt ist, ist für die oben aufgeführten Weiterverwendungen des Materials die Einwilligung des jeweiligen Rechteinhabers einzuholen.

Weitere Details zur Lizenz entnehmen Sie bitte der Lizenzinformation auf http://creativecommons.org/ licenses/by/4.0/deed.de.

\section{Literatur}

1. Arbab D, König DP (2016) Atraumaticfemoral head necrosis in adults. Dtsch Arztebl Int 113:31-38

2. Berlit $P$ (2010) Diagnosis and treatment of cerebral vasculitis. Ther Adv Neurol Disord 3:29-42

3. Birnbaum J, Hellmann DB (2009) Primary angiitis of the central nervous system. Arch Neurol 66:704-709

4. Citron BP, Halpern M, Mccarron M et al (1970) Necrotizing angiitis associated with drug abuse. NEngl J Med 283:1003-1011

5. Glick R, Hoying J, Cerullo L et al (1987) Phenylpropanolamine: an over-the-counter drug causing central nervous system vasculitis and intracerebral hemorrhage. Case report and review. Neurosurgery 20:969-974

6. Rumbaugh $\mathrm{CL}$, Bergeron RT, Scanlan RL et al (1971) Cerebral vascular changes secondary to amphetamine abuse in the experimental animal. Radiology 101:345-351

7. Deutsche Gesellschaft für Psychiatrie und Psychotherapie, Psychosomatik und Nervenheilkunde (DGPPN) (2016) S3-Leitlinie Methamphetaminbezogene Störungen

8. Deutsche Gesellschaft für Neurologie (2015) S3-Leitlinie Sekundärprophylaxe ischämischer Schlaganfall und transitorische ischämische Attacke

9. Sui B, Gao P (2019) High-resolution vessel wall magnetic resonance imaging of carotid and intracranial vessels. Acta Radiol 60:1329-1340 